\title{
Review of Recommendation System for Web Application
}

\author{
Preeti Bhole $^{1}$, Neetish Kumar Chandrakar ${ }^{2}$, Virendra Swarnkar ${ }^{3}$ \\ ${ }^{1}$ M.Tech Scholar, BCET, Durg, India \\ ${ }^{2,3}$ Assistant Professor, CSE, BCET, Durg, India
}

\begin{abstract}
Today's many number of users/customers that have no time for buying product in going market so users select on-line shopping for their interest much more, after studying papers it is clear that paper are designed for recommender system in big area. We will use A priory Algorithm and k-nearest neighbor algorithm. We will use techniques i.e. Association Rule Mining and Classification that are used to increase interest of users for recommender systems which is based on ratings of users in less timing.
\end{abstract}

Keywords: Recommender system, Association Rule Mining, Collaborative filtering, content based filtering, Classification

\section{Introduction}

\section{Recommendation System}

This system is developed by resnik and Varian in 1990.Recommendation system also called as "engine system". This system is used for filtering information that is used to predict the referencing (ratings) that a user would give to an item. Recommendation system have widely used in recent years and used in variety of area movies, music, books ,news, articals, social tags, research, online shopping etc. This system is used to suggest an item to the user and also provides users with information which any one may be interested in and give ratings via popularity. For example:Netflix, it's a video on demand providers in North America and UK.

There are mainly three types of Recommendation system Collaborative filtering Collaborative filtering model used to build model from users past record i.e. item previously purchased or Ratings given to those items. This model is used to rating items that user may have interest.

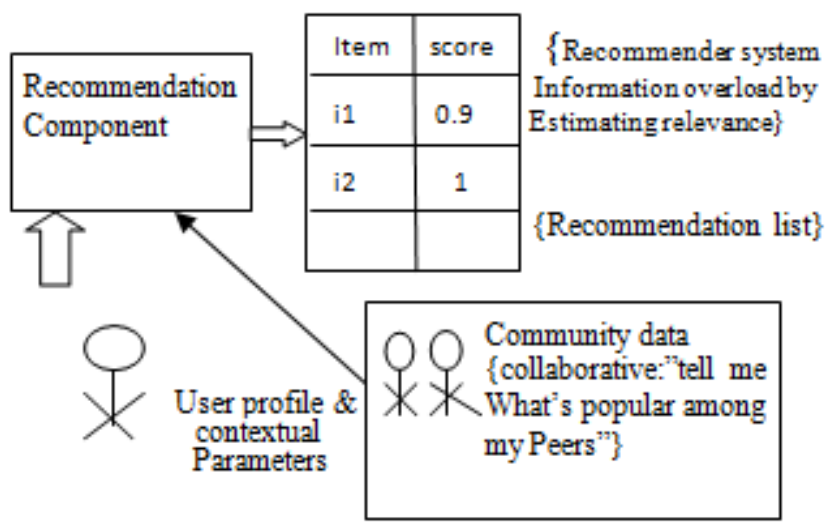

Figure 1.1: Collaborative filtering

There are again three types of collaborative filtering:-

Memory Based Collaborative filtering this approach uses rating data to compute the similarity between users and items. It is effective and easy to implement. This is used for making recommendations. This model is user based system.
Model based Collaborative Filtering this collaborative filtering is based on system model that are developed using data mining, machine learning algorithm used to find patterns on training data. These are used to make predictions for real data.

Hybrid Collaborative Filtering-This system is a combination of memory based and model based collaborative filtering system, it is used to improve prediction performance. They overcome the problem of CF such as sparsity, loss of information. Mostly commercial systems are hybrid Such as Google news recommendation system.

Content based filtering Content based filtering are based on description of the item and profile of the user reference item recommends to user based on table content for choosing an item.

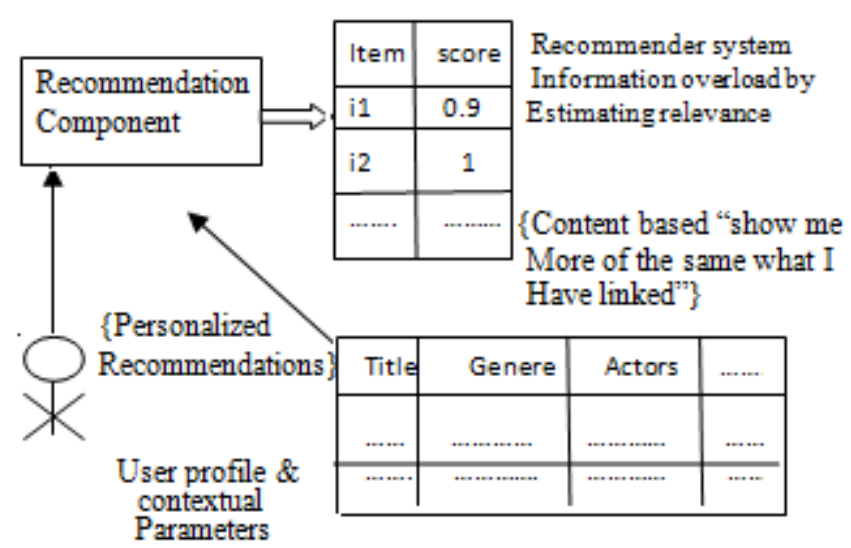

Figure 1.2: Content based filtering

Hybrid filtering this recommendation system is a combination of collaborative filtering and content based filtering system. And both are working together and reduced problem of each other. so most recommendation system are built in this concept. 


\section{International Journal of Science and Research (IJSR) \\ ISSN (Online): 2319-7064}

Index Copernicus Value (2015): 78.96 | Impact Factor (2015): 6.391

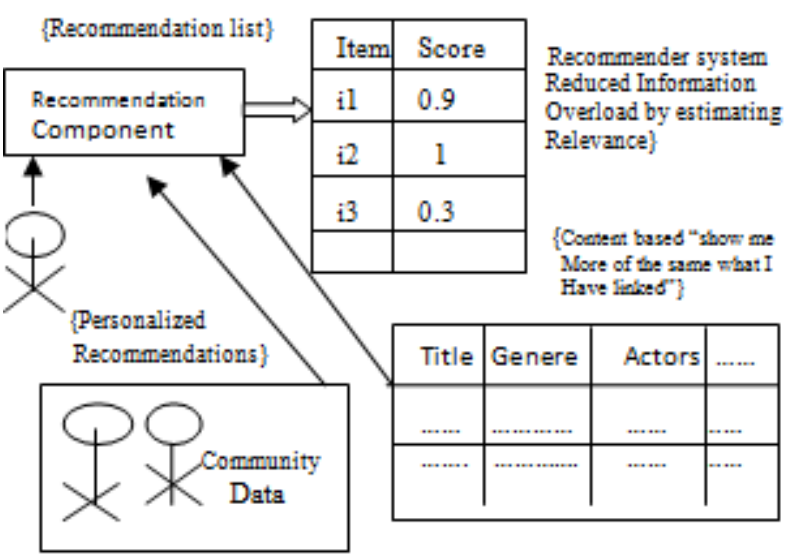

Collaborative $\quad$ Hybrids: - Combination of various inputs and/or composition of different Mechanisms\}

Figure 1.3: Hybrid Filtering

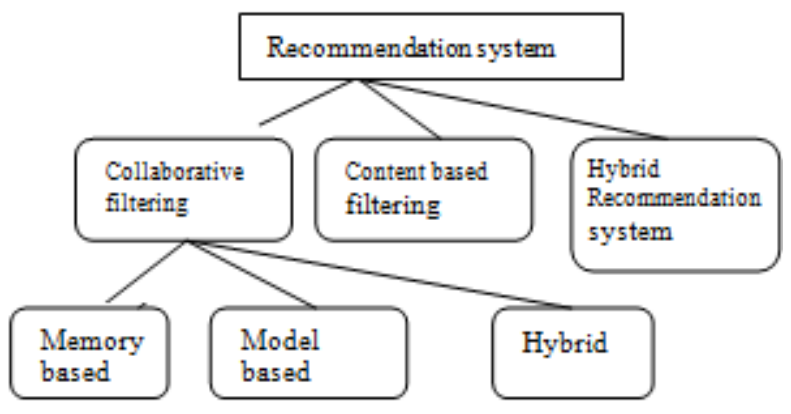

Figure 1.4: Classification of recommendation technique.

\section{Application of Recommendation System}

1) Online market place two things of market place where buyers and sellers sells item with each other.

2) Online news we can access news on line in recommendation system.

3) Online e-textbook we can learn self or grouped study with this sites. Also this is helpful for teachers.

4) Social and professional linking sites:-recommendation systems are used in social sites and peoples are read their opinion and give ratings like or dislike.

5) Online music suggestions:- recommendation system are also provide choosing movie online.

\section{Advantage of Recommendation System}

1) This system is used to improve the prediction performance.

2) This system increases number of items per orders.

3) This system is offer advice and direction for selecting item.

4) Recommendation system handles the problem of information overload.

5) Recommendation system used to improve decision making process and quality.

6) They reduced transaction cost of finding and selecting items in an online shopping.

7) Recommendation system is benefit to service provider and users.

8) This system is used for predict an preference an item not based on the users profile.

\section{Disadvantage of recommendation system-}

- Cold start:- when large amount of data are used to store and analyze in difficulty then system are slow, this problem are called cold start.

- Scalability-scalability means measurements/ calculations. If system has complexity for mining rules, then this problem is called scalability.

- Sparsity- when a product is very popular but ratings are low or almost zero then this problem are arising.

\section{Related Work}

Anand Shankar Tiwari at all (2014) has proposed Recommendation system are used to recommend books of user's interests. This paper based on content based and collaborative filtering and association rule mining.

Jennan Chen at all (2014) has worked on the design of recommendation system for small area venders and calculate small data pools and processing power. Also check accuracy and scalability of small area.

Sun Zhong at all (2013) has proposed personalized recommendation system of e-textbook use e-book platform for primary school between student and teacher for large class room.

Deuk Hee at all (2011) has proposed reviewed 164 articles on recommender system from 31 generals which were published from 2001 to 2009.the literature searches from which area of articles are most popular. In which 8 categories each of recommendation field and 8 categories each of mining techniques.

Chang-Ming Yan, Tzu-Jui Tang (2011) has worked to create a three tire architecture of recommender system i.e. customer tire, third party server tire and vender e-store tire based on the customers purpose. Which show customer detailed information for their privacy?

Akshita, Smita (2013) has proposed describe the area of recommender system to improve performance and also discuss about collaborative, mobile recommender system content based recommender system.

Amir Hussein at all (2013) has worked on overview of recommender system that includes collaborative filtering, content based filtering and hybrid system and also explain their weakness.

Shubham Shah at all (2016) has worked to present book recommender system which are select books for their buyers interest which is based on combining collaborative and content based filtering with Association rule .

Pranav Bhure, Navin Kumar Adhe (2015) has proposed to define book recommendation system that are give feedback comments from users and display top 10 books that are particular subject based on review feedback given by earlier people who read same book. Comments can be positive, negative and neutral one. 


\section{International Journal of Science and Research (IJSR) \\ ISSN (Online): 2319-7064 \\ Index Copernicus Value (2015): 78.96 | Impact Factor (2015): 6.391}

Neetish Kumar Chandrakar at all (2015) has worked on describe hybrid recommender system to proposed calculates the correspondence of users from their ratings. And also calculate unknown rating of a user to an item using a Book data set.

Raymond J .Mooney, Loriene Roy (1999) has worked to describe Content based book recommender system that utilized information extraction and machine learning algorithm for text categorization. This experiment is based on ratings.

RVVSV Prasad, V Valli Kumari (2012) has proposed to describe concept of recommendation system using collaborative, content based and Hybrid system and Agent recommendation system.

\section{Methodology}

\section{Association Rule Mining}

This technique is used for selecting an item in recommendation system. Association rule mining is used to find interesting rules among large data item. Assocaition rule are also used to identify what events occur before a failure in telecommunication. Association rules provide information in the form of "if-then" statements. "If" part are in left hand side and also called antecedent. "Then" part is in right hand side and called as consequent. Association rules are calculated by support and confidence.

Let $\mathrm{I}=\{\mathrm{i} 1, \mathrm{i} 2, \mathrm{i} 3 \ldots \mathrm{im}\}$ set of item.

Association rule can be represented by $A=>B$, where $A, B$ is a subset of $\mathrm{I}$, and $\mathrm{A} \cap \mathrm{B}=\varnothing$.Association rule eliminate the minimum support and minimum confidence.

Support- Support is number of transactions that include all items in the if then part. Support is sometimes express as a percentage of the total number of records in database. Support for association rule $\mathrm{X}=>\mathrm{Y}$. It is the percentage of transaction in database contains $\mathrm{X} U \mathrm{Y}$.

Confidence- confidence is the ratio of the number of transactions that include all items in the if-then part in transaction. Confidence is denoted by ' $c$ ', which represents the measure 'how often items in $\mathrm{Y}$ appear in transaction that contains X. Confidence denote strength of implication. Such rules with high confidence and strong support are referred to as strong rules. Task of association rule is to discover strong association rules in large database.

A priory Algorithm- A Priory algorithm is use large item set. It is bottom up search and moving upwards. This algorithm also called "the level wise algorithm". It is used to find all frequent set. At first iteration, a priory simply scans all the transactions to count the number of occurrences for each item. This algorithms are used to eliminate low support .and calculate accurate results.

Classification - Classification is the most popular data mining technique. This technique used to find rules that petition data. It is able to predict the class of objects whose class label is unknown such an image and pattern recognition, loan approval, detecting faults in industry applications etc.

K-nearest neighbors algorithms- these algorithms are used in distance measure in classification technique.KNN is assumes the entire training set including not only data in set but also desired classification for each item. Those item are selected which are mostly nearest for goal.

\section{Expected Conclusion}

Our project is based on recommender system that recommends an item in less timing and we will design a system for big area venders. To improve performance of system regarding scalability and timings.

\section{References}

[1] Anand shanker tiwari, abhey kumar, Asim Gopal Berman. "Book Recommendation System Based on Combine Features of Content Based Filtering, Collaborative Filtering and Association Rule Mining". IEEE, 2014, pp.500-50

[2] Jennan Chen, Courtney Miller, Gaby G. Dagher (2014). "Product recommendation system for small online retailers using Association rule mining “. IEEE 2014, pp. 71-77,

[3] Sun Zhong, Tian Xiaomeng, Shen Haijiao. "A research about personalized recommendatins in e-textbook". IEEE, 2013, pp.44-47.

[4] Deuk Hee Park, Hyea Kyeong Kim, I1 Young Choi, Jae Kyeong Kim. A Review and Classification of Recommender System Research .IPEDR, 2011, pp.V1290-294.

[5] Chang-Ming Yan, Tzu-Jui Tang. Apply CustomerCentered recommendation on an On-line Shopping System IEEE, 2011, pp.1993-1997.

[6] Akshita, Smita"Recommender System: Review". IJCA, 2013, pp. 38-42, vol 71-No.24.

[7] Amir Hossein Nabizadeh Refsanjani, Naomie Salim, Rezaei Aghdam, Karamollah Bagheri Fard. "Recommendation System: Review". IJCER, 2013, pp. 47-52, vol-03.

[8] Shubham shah, Aslam Motala, Niket Thombre, Charudutta Ugale, prof. P. S. Desai. "Book Recommendation System”. MJRET, 2016, pp. 977-981, vol-03.

[9] Pranav Bhure, Navinkumar Adhe. Book Recommendation System Using Mining Technique. IJRET, 2015, pp. 332-334, vol-04.

[10]Dr. Nitin Mishra, Neetish Chandrakar. Recommendation System For Online Shopping Based On Association Rules Mining Algorithm. IJRET, 2015.

[11]Kare Prashanthi, Nitin Mishra, Neetish kumar chandrakar, Saumya Chaturvedi. "Review of Various Recommendation Techniques for Web Applications". IJERT, 2015, pp. 133-136.

[12] Alexis Coulourides Kogan, Kathleen Wilber, Laura Mosqueda. "Person Center Care for Older Adults with Chronic Conditions and Functional Impairment: A Systematic Literature Review". JAGS, 2015, pp.1-7. 


\section{International Journal of Science and Research (IJSR) \\ ISSN (Online): 2319-7064}

Index Copernicus Value (2015): 78.96 | Impact Factor (2015): 6.391

[13] Raymond J. Mooney, Loriene Roy. "Content Based Book Recommending Using Learning for Text Categorization". SIGIR-99, 1999.

[14]RVVSV Prasad, V Valli Kumari. "A Categorical Review of Recommender System". IJDPS, 2012, pp. 73-83. 